\title{
A Systematic Review on Challenges Faced by Family Caregivers of Cancer Patients
}

\author{
Mehrnaz Keramatikerman* \\ Postgraduate Student in Master of Science in Nursing (Medical Surgical), Tehran University of Medical Sciences (TUMS), \\ Department of Nursing and Midwifery, Tehran, Iran Email: m-keramatik@razi.tums.ac.ir
}

\begin{abstract}
Objective: The cancer is one of the deadliest illnesses in the world which may last for a long period of time and require special medical attentions. The family of cancer patients as primary caregivers play a key role in taking care of the patient by spending long hours at home or hospital. They may suffer various challenges during their role as a caregiver which includes various challenges.

Methodology: A systematic review conducted on databases encompasses PubMed, Science Direct, Cochrane Library, Google Scholar from establish of database to by following the collective strategy of Cochrane Collaborative Group.

Results: A review on different challenges that are faced by family caregivers of cancer patients it was revealed that financial hardship, depression, isolation, emotional and physical burdens, time management, privacy insufficiency, and sleep deprivation. Whilst all challenges and associated difficulties have been reviewed and acknowledged in the literature, it has been revealed that less consideration has been paid on emotional burden as one of the key challenges for family caregivers.

Conclusion: The literature review revealed that there are no sufficient interventions conducted to ease difficulties occurred for the family caregivers during various cancer stages and/or after the patient death. Furthermore, it was also revealed that there is almost no support for caregivers to recover and adapt to the current condition. They may suffer from major depression many years and affect other member of family. Also, official supports from relevant authorities are recommended to form groups to follow up with families and to ease some of those challenges.
\end{abstract}

KEYWORDS: Family caregiver; Challenges; Cancer patient; Systematic review

\section{INTRODUCTION}

Cancer has been recognized as one of the most fatal diseases in the world which many people get involved each year [1]. Patients who are newly diagnosed with cancer, may face many unknown challenges such as physical and emotional problems that can impact on their quality of life and develop anxiety, depression and many more challenges [2-3]. Not only patients are dealing with healthrelated issues but also families play a key role in managing the illness as they spend most of the time and support clients at home [4]. The family caregiver is supposed to be an unpaid supportive individual who is providing medical and emotional help along with the diseases. They cope with the disease in every stage of cancer including diagnosing, treatment, monitoring, and patient followup [5]. One study has shown that one-fourth family caregivers of colorectal cancer patients face a variable range of stress during the diagnostic procedures [6]. The stress might be from unmet needs like expenses, emotional burden, side effects of chemotherapy and treatment process [7]. Probably the most family caregiver who is significantly affected by cancer is patient's spouses and experience

\section{Quick Response Code:}

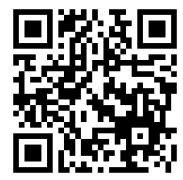

Address for correspondence: Mehrnaz Keramatikerman, Postgraduate Student in Master of Science in Nursing (Medical Surgical), Tehran University of Medical Sciences (TUMS), Department of Nursing and Midwifery, Iran

Email: m-keramatik@razi.tums.ac.ir

Received: June 30, 2020 Published: July 15, 2020

How to cite this article: Keramatikerman M. A Systematic Review on Challenges Faced by Family Caregivers of Cancer Patients. 2020 - 2(4) OAJBS.ID.000191. DOI: 10.38125/OAJBS.000191 
depression about 38.9\% during caring their loved ones [8]. Another study has shown that spending too much time on caregiving patients with cancer has a great impact on female spouses rather than male spouses as they hold more responsibilities at home beside the illness [9]. Family caregivers also have a fear of the cancer recurrence after survivorship and the level of strain distress is even higher than the patients and they are likely experience cardiovascular and infections effects from stress and poor immune system [10].

\section{Systematic Review Steps in Reviewing Literatures}

To understand the challenges faced by family caregivers of cancer patients, a systematic review was conducted as presented in a flow chart in Figure 1. As shown, all articles were identified through different databases and were screened two times due to duplications and being quite old and outdated. A full systematic review has been conducted on the remaining articles as can be seen in the flow chart.

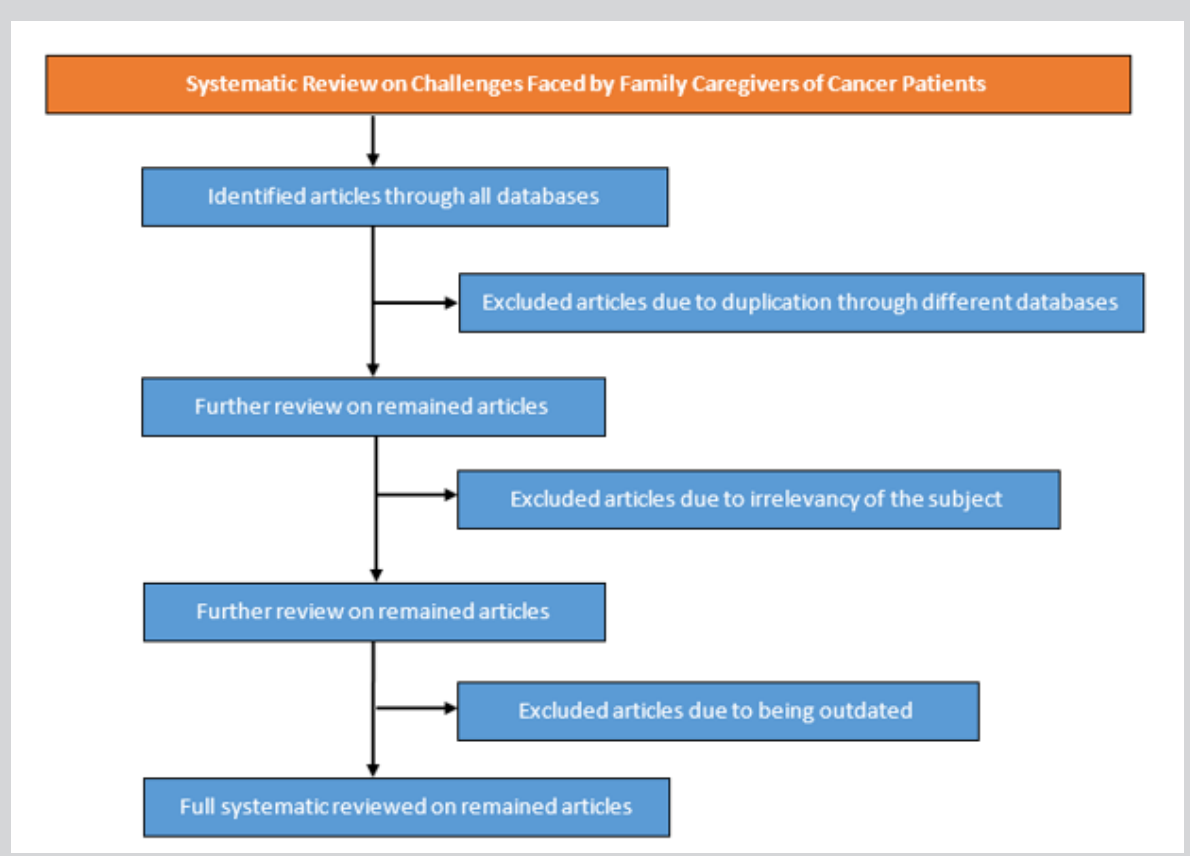

Figure 1: Flow chart followed to perform the systematic review in this study following the collective strategy of cochrane collaborative group.

On the other hand, healthcare professionals also overwhelmed with the patients as they are hospitalized for a long period of time for variety of reasons. Nurses are second caregivers who contact with cancer patients in hospital. They suffer from a diverse range of emotional problems such as drained of energy, losing mental balance after a death client or working in oncology ward for years [11].
A literature review conducted through different databases such as PubMed, Science Direct, Cochrane Library, and Google Scholar by following the collective strategy of Cochrane Collaborative Group showed that the main challenges that the family caregivers are financial hardship, depression, isolation, emotional burden, physical burden, time management, privacy insufficiency, and sleep deprivation. Figure 2 shows the diagram for the family caregiver challenges.

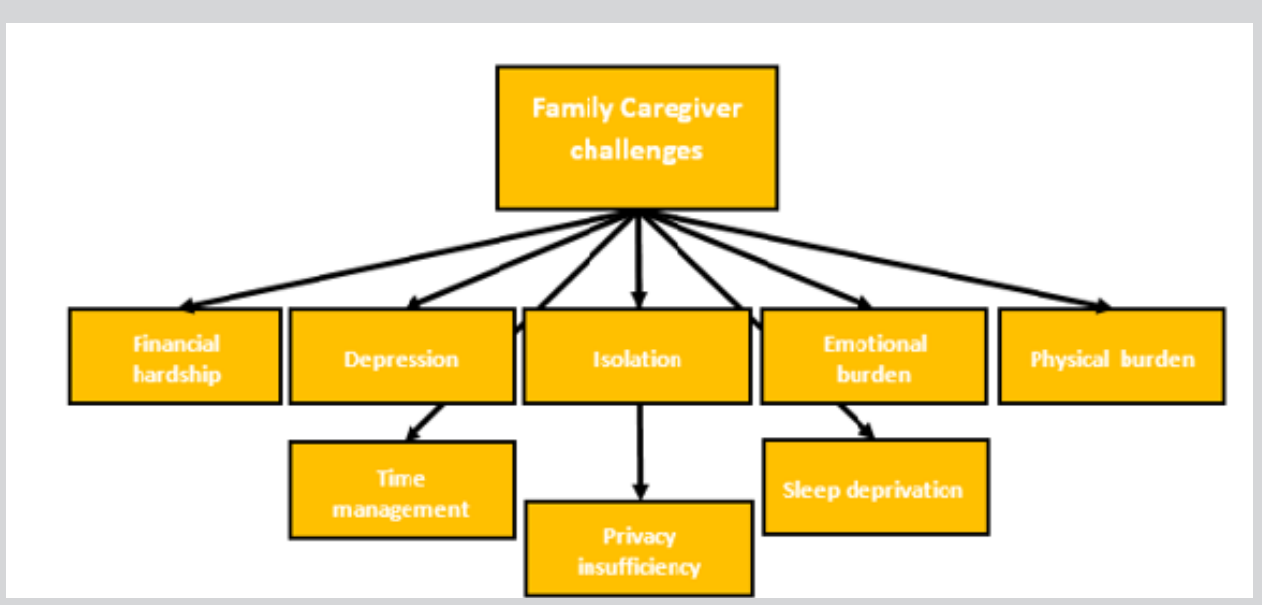

Figure 2: Diagram for main challenges for family caregivers of cancer patients. 
In the following sections some of the most challenges that are faced by the family caregivers have been discussed.

\section{Financial Hardship}

The financial strains or hardship has been indicated as one of the main challenges for the family caregiver challenges in the following studies [12-15]. It has been indicated that unpaid family caregiver often with a limited resource facing hourly basis costs as well as out of pocket expenditures have contribution to the health care system [13]. Another study conducted on challenges amongst family caregiver indicated that most of families of the patients are facing with severe financial constraints [14]. A review on the literature showed that families are facing with the cost of looking after their patient as well as their day to day financial requirements that impacts their quality of life and provides more concerns during the course of their role as a caregiver.

\section{Depression}

The depression is one of the key challenges that is faced by the family caregivers of cancer patients as indicated by many previous articles [16-19]. For instance, it has been indicated that the caregivers in the age range between 45 to 54 had the highest level of depression as they had an abandonment feeling [18]. As another example, the level of depression amongst family caregivers of cancer patient in Korea was in high level. The demographic studies shown that the spread of depression is more than $80 \%$ amongst family caregivers of the cancer patients in the Korea [20].

\section{Isolation}

Isolation is a common feeling amongst family caregiver of cancer patients that is occurred due to be far from family members and friends. The isolation sense and impact on the caregivers has been investigated in previous studies in a comprehensive [2124]. This feeling is happened when a family members, friends or acquaintances do not show any understanding or sympathy toward the process of the caring for a patient [22].

\section{Sleep Deprivation}

Family caregivers of cancer patients usually facing sleep disturbance due to their caring responsibilities. They sometimes required to be awake through night and address the requirements of their patients. Sleep disturbance or deprivation also has been one of the key areas of the studies in family caregiver of the cancer patients [25-30]. It has been indicated that the caregivers facing sleep impairments may be at the risk of physical and mental issues and may not be able to continue with their responsibilities if the sleep derivation in a short amount of time [31]. Other study indicated that the cancer patients and their family caregivers experiencing a same level of sleep interventions [32].

\section{Emotional Burden}

A review of the literature showed that most of previous studies investigated the impact of emotional burden in conjunction and in relation with the other challenges and not much standalone investigation conducted to investigate impact of emotional burden on family caregivers of cancel patients. For instance, it has been indicated that provision of the emotional support was amongst the most time taking and difficult tasks from family caregivers of the patients that receiving chemotherapy treatments [33]. Another study also indicated that there is mutual emotional relationship amongst the cancer patients and their family caregivers, however, does not indicated about the emotional burden on the family caregivers [34]. Other study also indicates that the presence of pain in a patient impact the family caregivers and put them in an anxiety and depression [35]. As seen, the reviewed literature for the emotional burden does not directly investigate the impact of emotional burden and this characteristic has been investigated amongst with other challenges.

\section{RESULTS AND ANALYSIS}

Family members are susceptible to various physical and emotional problems from initial to advance stage of cancer. They receive support from health care professions to alleviate the deep hardships during the hospitalization for diagnose, chemotherapy and palliative care. Nurses lead and support the informal caregivers in each condition. Few researches have been conducted to reduce the level of concerns in informal caregivers who constantly are in contact with patients. They well-being is precisely related to caring effectiveness and mental health as well. However, the importance of emotional burdens indirectly in some studies has been mentioned but rarely impact of the emotional burden as one of the key challenges is faced by the family caregivers of the cancer patients has been investigated.

\section{CONCLUSION}

A systematic review conducted on many articles amongst different databases such as PubMed, Science Direct, Cochrane Library and Google Scholar to investigate the challenges that are faced by family of the caregivers of the cancer patients. The results showed that the main challenges include but not limited to financial hardship, depression, anxiety, emotional burden, physical burden, isolation, time management, sleep deprivation, and privacy insufficiency. Whilst a review conducted on the main articles on some the main important challenges, it was revealed that emotional burden has been considered as a standalone challenge to the less extent in previous studies. It usually has been considered in conjunction with other challenges such as depression and anxiety.

\section{RECOMMENDATIONS FOR FUTURE STUDIES}

From the outcome of this study, it is recommended that the emotional burden as one of key challenges amongst the family caregivers of the cancer patients should be considered in the future studies.

\section{REFERENCES}

1. Sartor O, Loriaux DL (2006) The emotional burden of low-risk prostate cancer: proposal for a change in nomenclature. Clinical genitourinary cancer 5(1): 16-17.

2. Morrison EJ, Novotny PJ, Sloan JA, Yang P, Patten CA, et al. (2017) Emotional problems, quality of life and symptom burden in patients with lung cancer. Clinical Lung Cancer 18(5): 497-503.

3. Klapheke AK, Keegan TH, Ruskin R, Cress RD (2020) Changes in healthrelated quality of life in older women after diagnosis with gynecologic cancer. Gynecologic Oncology 156(2): 475-481.

4. Coluccia A, Ferretti F, Fagiolini A, Pozza A (2017) Psychometric properties and relations with coping and family strain of the Health Services and Caregiver Experience questionnaire (HSCE): an outcome measure of informal caregivers' experience for inpatient care in Italy. BMC health services research 17(1): 494.

5. Saimaldaher ZA, Wazqar DY (2019) Relationships between caregiving stress, mental health and physical health in family caregivers of adult patients with cancer: implications for nursing practice. Scandinavian Journal of Caring Sciences.

6. Mosher CE, Adams RN, Helft PR, O'Neil BH, et al. (2016) Family caregiving challenges in advanced colorectal cancer: patient and caregiver perspectives. Supportive Care in Cancer 24(5): 2017-2024. 
7. Blum K, Sherman DW (2010) Understanding the experience of caregivers: a focus on transitions. Seminars in oncology nursing 26(4): 243-258.

8. Braun M, Mikulincer M, Rydall A, Walsh A, Rodin G (2007) Hidden morbidity in cancer: spouse caregivers. Journal of clinical oncology 25(30): 4829-4834.

9. Li Q, Loke AY (2013) A spectrum of hidden morbidities among spousal caregivers for patients with cancer, and differences between the genders: A review of the literature. European Journal of Oncology Nursing 17(5): 578-587.

10. Northouse LL (2012) Helping patients and their family caregivers cope with cancer. In Oncology Nursing Forum 39(5): 500-506.

11. Zamanzadeh V, Valizadeh L, Sayadi L, Taleghani F, Howard F, et al. (2013) Emotional labour of caring for hematopoietic stem cell transplantation patients: iranian nurses' experiences. Asian Nursing Research 7(2): 9197.

12. Kim Y, Schulz R (2008) Family caregivers' strains: comparative analysis of cancer caregiving with dementia, diabetes, and frail elderly caregiving. Journal of aging and health 20(5): 483-503.

13. Northouse L, Williams AL, Given B, McCorkle R (2012) Psychosocial care for family caregivers of patients with cancer. Journal of Clinical Oncology 30(11): 1227-1234.

14. Rhee YS, Yun YH, Park S, Shin DO, Lee KM, et al. (2008) Depression in family caregivers of cancer patients: the feeling of burden as a predictor of depression. Journal of Clinical Oncology 26(36): 5890-5895.

15. Given BA, Given CW, Sherwood P (2012) The challenge of quality cancer care for family caregivers. In Seminars in oncology nursing 28(4): 205212.

16. Given CW, Stommel M, Given B, Osuch J, Kurtz ME, et al. (1993) The influence of cancer patients' symptoms and functional states on patients' depression and family caregivers' reaction and depression. Health psychology 12(4): 277

17. Kurtz ME, Kurtz JC, Given CW, Given BA (2004) Depression and physical health among family caregivers of geriatric patients with cancer-A longitudinal view. Medical Science Monitor 10(8): CR447-CR456.

18. Given B, Wyatt G, Given C, Gift A, Sherwood P, et al. (2004) Burden and depression among caregivers of patients with cancer at the end-of-life. In Oncology nursing forum 31(6): 1105

19. Carter PA (2003) Family caregivers' sleep loss and depression over time. Cancer nursing 26(4): 253-259.

20. Park B, Kim SY, Shin JY, Sanson-Fisher RW, Shin DW, et al. (2013) Prevalence and predictors of anxiety and depression among family caregivers of cancer patients: a nationwide survey of patient-family caregiver dyads in Korea. Supportive Care in Cancer 21(10): 2799-2807.
21. Stewart M, Barnfather A, Neufeld A, Warren S, Letourneau N (2006) Accessible support for family caregivers of seniors with chronic conditions: From isolation to inclusion. Canadian Journal on Aging/La Revue canadienne du vieillissement 25(2): 179-192.

22. Kristjanson LJ, Aoun S (2004) Palliative care for families: remembering the hidden patients. The Canadian Journal of Psychiatry 49(6): 359-365.

23. Ferrell B, Ervin K, Smith S, Marek T, Melancon C (2002) Family perspectives of ovarian cancer. Cancer practice 10(6): 269-276.

24. Given B, Given CW (1972) Patient and family caregiver reaction to new and recurrent breast cancer. Journal of the American Medical Women's Association 47(5): 201-216.

25. Carter PA (2002) Caregivers' descriptions of sleep changes and depressive symptoms. Oncol Nurs Forum 29(9):1277-83

26. Hearson B, McClement S (2007) Sleep disturbance in family caregivers of patients with advanced cancer. Interna J Pallia Nurs 13(10):495-501.

27. Fletcher BA, Dodd MJ, Schumacher KL, Christine Miaskowski RN (2008) Symptom experience of family caregivers of patients with cancer. Oncol Nurs Forum 35(2): E23.

28. Carter PA (2006) A brief behavioral sleep intervention for family caregivers of persons with cancer. Cancer nursing 29(2): 95-103.

29. Smith CE (1993) Quality of life in long-term total parenteral nutrition patients and their family caregivers. J Parenter Enteral Nutr 17(6): 501506.

30. Kim H, Rose K (2011) Sleep disturbances in family caregivers: an overview of the state of the science. Archives of Psychiatric Nursing 25(6): 456-468

31. Pawl JD, Lee SY, Clark PC, Sherwood PR (2013) Sleep characteristics of family caregivers of individuals with a primary malignant brain tumor. Oncology Nurs Forum 40(2):171-179.

32. Carney S, Koetters T, Cho M, West C, Paul SM, et al. (2011) Differences in sleep disturbance parameters between oncology outpatients and their family caregivers. Journal of clinical oncology 29(8): 1001.

33. Carey PJ, Oberst MT, McCubbin MA, Hughes SH (1991) Appraisal and caregiving burden in family members caring for patients receiving chemotherapy. Oncology Nurs Forum 18(8): 1341-1348.

34. Northouse LL, Katapodi MC, Schafenacker AM, Weiss D (2012) The impact of caregiving on the psychological well-being of family caregivers and cancer patients. Semin Oncol Nurs 28(4): 236-245.

35. Miaskowski C, Kragness L, Dibble S, Wallhagen M (1997) Differences in mood states, health status, and caregiver strain between family caregivers of oncology outpatients with and without cancer-related pain. Journal of Pain and Symptom Management 13(3): 138-147. 